\title{
Digital radiography detectors: a technical overview
}

\author{
Luís Lança and Augusto Silva
}

\begin{abstract}
Developments in digital detector technologies have been taking place and new digital technologies are available for clinical practice. This chapter is intended to give a technical state-of-the-art overview about computed radiography (CR) and digital radiography (DR) detectors. CR systems use storage-phosphor image plates with a separate image readout process and DR technology converts $\mathrm{X}$-rays into electrical charges by means of a readout process using TFT arrays. Digital detectors offer several advantages when compared to analogue detectors. The knowledge about digital detector technology for use in plain radiograph examinations is thus a fundamental topic to be acquired by radiology professionals and students. In this chapter an overview of digital radiography systems (both CR and DR) currently available for clinical practice is provided.
\end{abstract}

Keywords: Computed radiography; Digital radiography; Detectors; Storage-phosphor image plates; Image readout process; Electrical charges; X-ray; Readout process; TFT arrays; Analogue detector; Plain radiograph; Clinical practice. 